\title{
Du paradis à Dream Park, les jardins dans le monde arabe : \\ Damas, Le Caire, Rabat
}

\author{
From Paradise to Dream Park, gardens in the Arab world: \\ Damascus, Cairo, Rabat
}

Paru dans Annales de Géographie n650, 2006, p.409-433

\author{
Gaëlle Gillot \\ MCF IEDES-Paris 1
}

\begin{abstract}
Résumé :
Évocations du paradis sur terre, les jardins arabo-musulmans, aménagés selon des règles relativement précises, ont voyagé et ont fécondé l'imaginaire de toute une région, et de l'Occident, tout en étant un élément constitutif et caractéristique des villes de cette aire géographique. Ils correspondent à une sorte d'âge d'or de la civilisation arabo-musulmane. $\mathrm{Au} 19^{\text {ème }}$ siècle, un mouvement de modernisation des villes, soit recherché par les pouvoirs locaux soit imposé par les colonisateurs a introduit le jardin public et a induit dans l'aménagement urbain une rupture franche avec la tradition paysagère arabe. La représentation occidentale de la ville, hygiéniste et donc riche en jardins publics, est devenue le canon de la ville moderne. Ayant perdu leur symbolique de modernité pendant les années de croissance incontrôlée des villes, les jardins publics ont été abandonnés pour redevenir depuis les années 1970-1980, sous la forme d'espaces verts de nouvelles panacées aux maux urbains. Au cours de cette mutation de vocabulaire, ils ont également perdu leur sens d'espace public pour devenir des jardins dévoués aux distractions et ont été graduellement privatisés, ce qui a favorisé l'émergence de nouveaux types de lieux de loisirs, suréquipés, payants et sur le modèle de Disney Land. Ces trois phases des jardins correspondent à trois époques et à trois types de sociétés, et symbolisent une longue marche vers la perte de la spécificité arabe des villes.
\end{abstract}

\begin{abstract}
:
Evocation of Paradise on earth, Arab and Islamic gardens, organised according to welldefined precise rules, have reached the entire Western world and fueled its imagination; it has also been a distinctive element of the cities in the Arab word. They are representative of a kind of "golden age" of the Arab civilisation. At the $19^{\text {th }}$ century, a modernising movement in the cities, either wished or imposed by the colonizers, induced the public garden and induced in the urban organisation a clear rupture with the Arab landscaping tradition. The Western representation of the city, hygienist and rich of public gardens, became the model of a modern city. Having lost their modernity symbolic during the period of the enormous growth of the cities, public gardens were abandoned. Around the 1970 's/1980's, they made a come back as green areas, new remedy for urban diseases. In this mutation of the vocabulary, they also lost their meaning as public space to become places for leisure time; it went along the emergence of new types of leisure spaces, with a lot of equipments, paying, following the example of DisneyLand's park concept. Those three stages in garden's evolution correspond to three periods and three types of societies and symbolise a long lasting process of lost of Arabic specificity of the cities.
\end{abstract}

Mots-clés : jardin arabo-islamique, représentation de la ville, jardin public, modernisation, idéal urbain, hygiénisme, Le Caire, Rabat, Damas. 
Key-words : Arabo-Islamic garden, representation of the city, public garden, modernisation, ideal city, hygienism, Cairo, Rabat, Damascus.

Le jardin est un objet singulier qui peut en dire long sur les sociétés, leurs représentations et réalisations. Dans le monde arabe, il renvoie de façon inévitable à Babylone, à l'Alhambra ou encore aux lieux secrets du Bagdad des Mille et Une Nuits. Il évoque immanquablement le rêve d'un espace paisible et secret. Ces jardins-là, clos et privés, ont structuré les villes arabes et leur ont donné une forte identité paysagère et culturelle. Mais ils appartiennent désormais à l'histoire et au patrimoine. S'ils ont été des modèles, ils ne le sont pas demeuré dans des villes, telles que Rabat, Damas ou Le Caire qui ont profondément changé depuis l'époque mythique des Abbassides ou des Omeyyades, et ont adopté de nouveaux modèles urbains, de gré ou de force.

Face à l'histoire millénaire des jardins privés de l'Orient arabe, les jardins publics n'ont qu'une histoire récente à opposer. Elle est liée aux échanges culturels et idéologiques entre l'Europe - et en particulier la France - et le monde arabe, mais aussi à l'histoire coloniale du bassin méditerranéen. Le jardin public est né en Europe et a pris son sens contemporain notamment à Paris sous le Second Empire. Il a été exporté dans le monde arabe, et son influence sur les tissus urbains provient de son lien avec la fondation d'un espace public urbain, et ne peut être distingué du mouvement urbanistique moderne qui a modifié les façons de se représenter et de faire la ville, de même que les références utilisées.

Les transformations du jardin sont révélatrices de l'évolution de la ville, qui elle-même informe sur l'époque et le type de société. Dans le monde arabe, les modifications de formes et d'esprit de ces espaces rendent compte du passage d'une «ville arabe » à une ville fortement marquée par une modernité à l'Occidentale. Les jardins publics du monde arabe sont aujourd'hui davantage inspirés par Disney Land que par l'Alhambra. De l'imaginaire au spectacle commercial, des jardins antiques rêvés aux nouvelles formes de parcs de loisirs, la mutation de la ville arabe peut se lire 
à travers celle de ses jardins qui éclaire une dynamique des formes et des représentations de la ville au fil du temps.

Elles se rattachent à un mouvement général de «correction» des identités régionales qui met en lumière une circulation des modèles et une perte de spécificité. Nous l'analyserons à partir de l'étude de la charge imaginaire des jardins mythiques de l'Orient arabe, des bouleversements urbains de l'époque coloniale, et en cherchant à dégager la tendance récente des jardins dans ces trois villes ${ }^{1}$. Du jardin-paradis, au jardin-distraction en passant par le jardin-hygiéniste, la forme des espaces raconte trois époques, trois conceptions des jardins et trois types de sociétés.

\section{I) Les jardins de l'imaginaire}

\section{1) Les jardins fondateurs. Mythes, paradis, et âge d'or de la civilisation arabo-islamique}

«À l'extrémité de ce salon, (...), de grandes fenêtres ouvraient sur l'extérieur: basses elles permettaient au regard de tout voir d'un riche jardin dont le massif de fleurs reproduisait en écho les dessins de la coupole. Sur les côtés, l'eau se déversait d'un grand bassin dans un autre, plus petit et, au bord, dans des vases de métal plaqué d'un or que rehaussaient des pierres d'un grand prix, poussaient $d u$ basilic, des nénuphars, des narcisses, des roses de toutes les couleurs, des violettes, des camomilles, des giroflées rouges. Les arbres avaient, tout autour, mêlé leurs branchages et laissaient pendre leurs fruits mûrs. On pouvait voir ceux-ci se détacher pour venir doucement s'abandonner sur la surface de pièces d'eau (...). C'est que nombreuses étaient les espèces volantes, qui du haut du ciel étaient attirées par ce jardin, s'y laissant guider d'un battement d'ailes, et là,

\footnotetext{
${ }^{1}$ Cet article a été écrit à partir d'une thèse de doctorat en géographie « Les jardins publics dans les grandes villes du monde arabe. Politiques et pratiques au Caire à Rabat et à Damas », soutenue à l’Université de Tours en 2002.
} 
se livraient à des chants alternés composant une sorte de conversation ininterrompue $»^{2}$.

Vrais ou imaginés, les jardins de l'Orient arabe possèdent tous les mêmes caractères clés. Dans cette description d'un jardin de Bagdad à l'époque du calife Haroun el-Rachid, tous les éléments du jardin idéal sont représentés. Ils provoquent le rêve et la fascination. Seule manque la description de la géométrie et des mosaïques pour correspondre totalement au «modèle » du jardin arabe, fondé à la fois sur l'héritage perse et les descriptions du Coran qui l'assimile au paradis.

Dans l'histoire humaine, les très anciens témoignages de jardins sont considérés comme un premier signe de civilisation de l'homme sédentaire. Le jardin se trouve en effet à la frontière de la nature et de la société. Il semble que ce soit en Mésopotamie que l'on ait retrouvé les vestiges des plus vieux, aux alentours du troisième millénaire avant $\mathrm{J}-\mathrm{C}$. Les jardins les plus célèbres de cette région restent ceux de Babylone, archétypes de l'œuvre qui pérennise une civilisation. Classés par le Grec Antipatros de Sidon parmi les Sept Merveilles du monde antique, ils sont devenus une légende. Ils restent cependant une référence incontestable de l'influence des jardins de la Mésopotamie en Grèce, à Rome et plus tard dans le monde arabe à l'époque abbasside et celle de la rapide extension de l'islam. Ni leur localisation ni leur date de construction n'est sûre, mais rien ne permet de mettre leur existence en doute, d'autant que la légende de leur édification leur procure une plus-value émotionnelle et romantique incontestable. Selon l'historien Flavius Josèphe, ils auraient été construits sous le règne de Nabuchodonosor II (604-562 av. J-C). La petite histoire ajoute que ce serait par amour de son épouse d'origine perse, la célèbre reine Sémiramis, qui regrettait les collines et les montagnes boisées de son pays, que le souverain aurait fait aménager ces jardins. Leur système hydraulique ingénieux et complexe les distinguait particulièrement, et situés là où le Tigre et l'Euphrate sont les plus proches, ils pouvaient bénéficier d'eau en abondance dans une région pourtant aride. Ils se présentaient sous la forme

\footnotetext{
${ }^{2}$ Les Mille et une nuits, (traduction de René Khawam), tome II : « Les cœurs inhumains », édition Phébus, Paris, 1989, 366 p., p. 278.
} 
d'un ensemble de terrasses sur lesquelles des arbres et des fleurs étaient plantés, donnant une image de fertilité et rehaussant la majesté des bâtiments environnants.

Ainsi, les jardins de Babylone sont devenus un mythe fondateur dans l'imaginaire des jardins, alors qu'en Perse se développaient des jardins d'agrément moins spectaculaires, mais dont l'influence fut très importante : les paradeisos. L'exemple le plus connu est le jardin du palais de Pasargades, à environ cent kilomètres au nord de l'actuelle Chiraz (Iran). Conçu selon un plan que l'on appelle chahar bagh (ou tchahar bagh), le jardin perse se présentait sous la forme d'un rectangle clos subdivisé par des canaux d'irrigation en quatre rectangles égaux. Les Perses qui se disaient «maîtres des quatre quartiers du monde» adoptèrent cette simplicité géométrique que reprennent plus tard les musulmans. Les jardins d'agrément se sont répandus dans le monde antique à partir de la Perse par les conquêtes, les empires, les échanges de toute forme dans un mouvement de « fertilisation culturelle croisée $»^{3}$. Ils voyagèrent en Syrie et en Égypte, jusqu'à l'Espagne et au Maghreb, influençant la Grèce et l'Italie romaine.

De façon universelle, l'homme a fait de cet espace clos une représentation du monde et de la nature. Il a voulu qu'elle soit parfaite et donc extrêmement belle, agréable, rafraîchissante, parfumée, reposante et surtout plus facile à vivre que le milieu naturel dans lequel il évoluait quotidiennement. La nature magnifiée a ainsi été assimilée au paradis, « idée compliquée (...), avant ou après la vie, mais jamais pendant; une illusion qui n'est nulle part spatialisée, mais où la nature est magnifique ${ }^{4} »$. Or, le paradis décrit dans les livres religieux est assimilé à des jardins merveilleux. Elle représente un monde idéal dans lequel la vie elle-même serait idéale.

Dans les pays de désert où l'islam est né, la nature est visiblement hostile aux hommes. L'eau est la denrée la plus précieuse et en maîtriser le stock et l'usage est la seule condition de survie. Les caravanes ne pouvaient trouver le repos que dans les oasis, synonymes d'eau, de verdure, d'ombre, de fraîcheur, d'abondance, de repos. Elles se présentent ainsi comme des

\footnotetext{
${ }^{3}$ Selon l'expression de John Brookes, Gardens of paradise. The history and design of the great Islamic gardens, London, Weidenfeld and Nicholson, 1987, p. 32

${ }^{4}$ Séverine Auffret, Aspects du Paradis, Arléa, 2001, (introduction).
} 
antithèses du désert. L'eau en constitue l'âme et sa présence rend l'existence de l'oasis «miraculeuse ». On la désire donc abondante et facilement repérable alors qu'elle est rare. L'oasis tranche par toutes ses caractéristiques sur le désert: féconde comme les femmes, elle nourrit, accueille, réconforte ; elle comble les sens malmenés par les rigueurs de la chaleur et de l'aridité. Son environnement est perçu alors comme idéal et inattendu, comme un cadeau de la nature, ou de Dieu. Dans ces conditions, il n'est pas étonnant que l'oasis soit considérée comme la référence première des jardins orientaux.

Ainsi, le jardin de l'islam est héritier de la tradition perse. L'étymologie et la tradition ont réutilisé facilement le plan d'ensemble géométrique et les quatre fleuves qui selon le Coran coulent au paradis. L'islam est né dans un pays où, écrit Michel Baridon (p. 210), «tout prédispose l'homme à apprécier les bienfaits de la nature, car elle n'en est guère prodigue ». Si l'oasis est la référence générique du paradis décrit par le Coran, il semble que Damas ait constitué un modèle de choix pour sa description. Selon la Tradition, le Prophète n'a-t-il pas refusé d'entrer à Damas sous le prétexte qu'on ne pouvait pénétrer au paradis qu'une seule fois? Dans Gardens of Paradise. The history and design of the great Islamic gardens, John Brooks écrit que «les descriptions des jardins du Coran ont pu être inspirées par les réels jardins de Damas, ville qui aurait été vue et appréciée par les marchands de la Mecque à la fin de leur longue route caravanière et qui auraient été vus comme un véritable paradis en contraste à l'aride sauvagerie de leur pays d'origine » (p. 19). Les jardins de Damas représentaient ainsi une sorte de paradis sur terre, un monde meilleur dans lequel on pouvait pénétrer sans qu'il soit nécessaire de mourir.

De fait, c'est de façon insistante que reviennent dans le Coran les évocations d'une vie bienheureuse dans une nature extraordinaire. On note à la suite d'André Miquel $^{5}$ que le mot djanna, qui signifie à la fois paradis et jardin en arabe, $\mathbf{y}$ est utilisé plus de deux cents fois et qu'il y a un peu plus d'une centaine de versets consacrés à la notion de paradis.

\footnotetext{
${ }^{5}$ La géographie du monde musulman, Paris, La Haye, New-York, Mouton (éditions de l'EHESS), 1980, t. 3
} 
Louis Gardet, dans son article «Djanna» de l'Encyclopédie de l'islam décrit quelques uns des plaisirs du paradis énumérés dans le Coran. Les jardins seront sublimes (LXXXVIII, 10) et garnis de nombreuses fontaines à l'eau jaillissante, fontaines aromatisées de camphre ou de gingembre (LXXXVI, 5 et 17). Ils seront parcourus par des ruisseaux d'une eau vive (LXXXVIII, 10). L'ombrage des arbres magnifiques, rares et généreux sera large et étendu. On y trouvera des fruits à profusion et à toute saison. Les épines des végétaux n'existeront plus. Des pavillons s'y trouveront, occupés par des vierges (les fameuses houris), prêtes à accueillir les élus. Des parterres de plantes belles et précieuses seront séparés les uns des autres par des canaux d'eau fraîche. Des chemins tracés en suivant les axes du jardin permettront de se promener dans ce paysage idéal.

Il parait logique, face à ces descriptions précises parsemées de détails sensibles le rendant «réalisable», que les hommes aient tenté de faire descendre le paradis sur terre en créant des jardins dans les villes.

Damas était sans doute la ville inspiratrice des descriptions du paradis du Coran, mais d'autres cités de la terre d'islam, par la réalisation de jardins, restent des références culturelles et paysagères. Damas, Marrakech et Bagdad notamment possèdent dans l'imaginaire populaire une place à part et une postérité qu'elles doivent à la présence de jardins devenus de vraies légendes. Mais plus encore que ces villes, Grenade reste inégalée et représente l'apogée d'une civilisation qui décline pourtant rapidement à partir du $11^{\text {ème }}$ siècle.

Quand Abd al-Rahman $1^{\text {er }}$, petit fils du calife de Damas, qui avait échappé au massacre de sa famille en 750, arriva en Espagne, il fit de Cordoue la capitale de son émirat. Il y fonda la dynastie des Omeyyades, semant ainsi les graines du «mythe andalou ». Sous Abd al-Rahman III de grands jardins furent aménagés dans une nouvelle ville, Madinat al-Zahrâ, près de Cordoue. Le califat de Cordoue était alors «l'ornement du monde ${ }^{6} »$. C'est à Grenade cependant, seule rescapée de la conquête arabe et dominée dès lors par les Almohades venus du Maroc, que l'art des jardins fut porté à son apogée au cours du $13^{\text {ème }}$ siècle. L'Alhambra et le Generalife sont aujourd'hui encore considérés comme les plus somptueux jardins de

\footnotetext{
${ }^{6}$ Michel Baridon, op. cit., p. 244
} 
l'aire arabo-musulmane. Ils sont le fruit d'un croisement de connaissances venues d'Orient, d'influences venues du Maroc et issues du bouillonnement intellectuel qui caractérisait Grenade à cette époque de résistance à la Reconquête chrétienne. Conçus dans le but d'être une «pure louange à Dieu et au sultan, un desiderata réitéré de béatitude et de bonheur perpétuels ${ }^{7} »$, les jardins de l'Alhambra et du Generalife se référaient à une conception modèle de la cité, une ville paradis.

Ainsi, les références des jardins arabo-islamiques puisent leurs sources dans la Perse et la Mésopotamie antiques pour devenir des lieux de nature idéale, qui répondent à certaines règles : l'abondance en eau et en végétation, l'organisation géométrique et l'usage des couleurs, la splendeur. Par définition, tout comme l'oasis est clairement délimitée, ces jardins sont clos, et cela d'autant plus qu'ils sont contenus la plupart du temps dans les cours intérieures des habitations. Espaces privés, retraites, ils peuvent alors être un lieu de relaxation, hors du monde et du temps.

On retrouve ces caractéristiques, devenues génériques, dans la plupart des jardins privés de la région. Elles représentent un marqueur culturel. Fascinante, cette conception des jardins a des conséquences sur la représentation de l'Orient arabe par les Occidentaux, et contribue à mettre en place le « rêve oriental » à partir de la fin du $18^{\text {ème }}$ siècle. La réalisation de jardins aux références arabo-islamiques par les Européens dans le cadre de la colonisation et de la construction des nouvelles villes, est une manifestation de la force de cette culture et de la spécificité de l'organisation physique au sein de la ville arabe.

La structure des jardins privés a aussi une répercussion sur la forme de la ville arabe qui a grandi avec ou contre ses jardins. Les jardins d'agrément « à l'andalouse », n'étaient pas aussi répandus que l'on peut le penser dans toutes les villes du monde arabe. Beaucoup de ceux qui ont donné lieu à une description des poètes ou des voyageurs émerveillés étaient pour beaucoup les jardins maraîchers ou les vergers qui entouraient les villes, les nourrissaient et leur procuraient des lieux de promenade.

\footnotetext{
${ }^{7}$ José Miguel Puerta Vilchez, «L'architecture parlante », Qantara, n³7, automne 2000, p. 42.
} 
1.2) Des jardins dans et autour de la ville: un trait commun.

À défaut de définir avec précision un modèle unique de ville araboislamique, ce qui n'est d'ailleurs pas l'objet ici, on peut remarquer dans les villes de l'aire arabo-islamique, certains traits communs aux agglomérations qui ont marqué une spécificité, au-delà des discontinuités régionales, des différences dans les développements historiques et politiques et de la variété des conditions climatiques et topographiques. La ville dans la pensée arabe médiévale représentait avant tout une qualité de $v^{2}{ }^{8}$. La vie urbaine était considérée comme supérieure au milieu non urbain grâce aux qualités esthétiques et hédonistes qu'elle permettait. La subtilité des arts et le progrès des sciences étaient des manifestations de la puissance du prince qui déployait alors les moyens nécessaires à faire de sa ville une cité prospère et raffinée. Les plans à cour (sur le modèle de la maison du Prophète à Médine) des lieux de culte, de l'habitat et des édifices à fonction commerciale étaient dominants, même s'ils n'étaient pas uniques'. Ils ont permis l'aménagement de jardins avec fontaines dans les habitations, essentiellement celles des groupes aisés, car ils nécessitaient la propriété de grandes parcelles.

Le besoin de se nourrir pour la ville fortifiée a induit la construction de larges enceintes (surtout à partir du $10^{\text {ème }}$ siècle) afin de permettre les cultures à l'intérieur des murs. Ainsi, bien qu'elle eut un aspect très minéral en raison de l'introversion des bâtiments, des ruelles étroites sinueuses et aveugles, la ville ne rompait pas le lien avec la nature comme il est recommandé dans le Coran. Elle était entourée de plantations qui, outre le ravitaillement, assuraient le lien et la transition avec l'environnement non urbain ainsi qu'une partie spirituelle de ses qualités esthétiques. Bien que la

\footnotetext{
${ }^{8}$ Doris Behrens-Abouseif, « La conception de la ville dans la pensée arabe du Moyen Age », dans Nicolet, Ilbert, Depaule (dir.), Mégapoles méditerranéennes. Géographie restrospective, Paris, Maisonneuve \& Larose, MMSH, Ecole française de Rome, 2000, p. 32-40.

${ }^{9}$ Voir Sylvie Denoix, « Unique modèle ou type divers? La structure des villes du monde arabo-musulman à l'époque médiévale », dans Nicolet, Ilbert, Depaule (dir.), Mégapoles méditerranéennes. Géographie restrospective, Paris, Maisonneuve \& Larose, MMSH, Ecole française de Rome, 2000, p. 912-937
} 
projection du Paradis sur terre soit un élément d'explication à ces jardins, il n'est bien entendu pas suffisant. Ainsi, une cité réussie était un lieu où l'on pouvait, en plus de la justice et du commerce, jouir des arbres, des oiseaux, des sucreries, de musique, de vin et même de hachich, ce qui contribuait à la joie de vivre $^{10}$. Or ces éléments sont profondément caractéristiques des jardins d'agrément d'alors.

Ainsi organisés (dans et autour de la ville) ils font partie des traits communs spécifiques à la cité arabo-islamique et contribuent à la structurer. Si l'on s'attache à la place de la religion dans ces villes, on perçoit également qu'en vertu d'une gestion raisonnée de l'eau, les jardins sont un des trois éléments clés ${ }^{11}$ de la ville islamique. En effet, la ville noyau s'organise autour de la grande mosquée (ou du palais). La mosquée doit posséder un puits afin de procurer l'eau nécessaire aux ablutions des croyants. Des bains attenants à la grande mosquée au moins (nécessaires aux grandes ablutions du vendredi) ont également besoin d'approvisionnement en eau par un puits. Or, dans ces régions arides, l'eau devait être utilisée avec parcimonie. C'est ainsi qu'elle était récupérée dans un réservoir (birka) souvent commun à la mosquée et aux bains afin, une fois plein, de servir à l'irrigation de jardins (maraîchers, ou vergers, voire plus rarement d'agrément) aménagés sur des parcelles contiguës à la mosquée et aux bains. Cette organisation spatiale dictée à la fois par les besoins de la religion et de l'eau a profondément marqué les villes araboislamiques. On en trouve encore la trace dans la vieille ville de Sanaa dont les jardins maraîchers, cachés derrière de grands murs sont invisibles de la rue, mais ont contribué à la survie au développement et au rayonnement de la cité et occupent encore une grande partie de la surface de la vieille ville ${ }^{12}$.

Ainsi, la cité arabo-islamique possède un rapport aux jardins à la fois idéel et pratique, qui entre autres définit un système urbain cohérent, homogène, jusque dans ses extensions. Celles-ci ont partout été réalisées par

\footnotetext{
${ }^{10}$ Doris Behrens-Abouseif, « La conception de la ville dans la pensée arabe du Moyen Age », op. cit.

${ }^{11}$ La mosquée principale ou le palais, les bains, les jardins.

${ }^{12}$ Voir à ce propos Barcelo Miquel, Gillot Gaëlle, Marquez Maite, Pelat Frédéric, Piera Arcadi, Les jardins de la vieille ville de Sanaa, Centre Français d'Archéologie et de Sciences Sociales de Sanaa, Universitat Autonoma de Barcelona, Servei de Publicacions, Bellaterra, mars 2004
} 
densification du tissu au détriment des espaces libres tels que les jardins intérieurs, et par élargissement de l'aire urbaine sur les vergers extérieurs. Les jardins sont souvent considérés comme des « éléments accessoires » ${ }^{13}$. Ils ont pourtant révélé un art de vivre urbain, une organisation écologique et une qualité esthétique qui ont permis d'affirmer une appartenance de ces villes à une même aire culturelle, et à assurer leur postérité et leur légende. Certaines villes comme Damas, Marrakech, Bagdad, Grenade se distinguent particulièrement des autres par leur « génie hydraulique » qui leur a permis de grandir et de se maintenir.

Damas a été fondée dans une oasis, la Ghouta, particulièrement verdoyante et fertile, grâce au petit fleuve Barada qui la traverse. Pour la développer, il a fallu réaliser un système de canaux de distribution de l'eau à partir du fleuve. Rendre l'eau abondante alors qu'elle est rare est un signe de la supériorité de la civilisation sur la nature. C'est ainsi que dans les palais et toute belle demeure on installait des fontaines dans les cours intérieures. La végétation permettait de protéger les demeures de l'excessive chaleur de l'été. On cultivait autour de Damas de très nombreuses espèces d'arbres fruitiers entre lesquels des légumes poussaient facilement et en abondance ainsi que la très réputée rose de Damas. C'est cette eau fraîche et apparemment abondante, jaillissant des fontaines, les vergers touffus et les riches cultures maraîchères côtoyant des palais raffinés qui ont donné à Damas une esthétique idéale et un sentiment de paradis.

Bagdad, devenue capitale du calife abbasside Al-Mansur (754-775), possédait l'avantage d'être située entre le Tigre et l'Euphrate. Les travaux d'hydraulique n'avaient jamais encore atteint l'ampleur qu'ils prirent alors. Toute la zone entre les deux fleuves était couverte de canaux d'irrigation, démontrant le pouvoir du prince à rendre fertile le désert. La ville avait été voulue somptueuse et sa forme circulaire donnée par Al-Mansur est restée célèbre. Des jardins avaient été aménagés autour du palais, situé au centre exact de la ville, et étaient pourvus de nombreux jeux d'eau, d'arbres fruitiers et de riches kiosques, accentuant l'impression de luxe. Bagdad est considéré pour cette période comme une des plus belles cités de l'empire

\footnotetext{
${ }^{13}$ Jean-Claude Garcin, « Le moment islamique (VIIe-XVIIIe siècles) », dans Nicolet, Ilbert, Depaule (dir.), Mégapoles méditerranéennes. Géographie restrospective, op. cit., p. 90-103
} 
abbasside, comme une réalisation idyllique synthétique de la ville, inspirée par Dieu, et le calife.

Marrakech fait partie de ces villes légendaires, renommées pour leur douceur de vivre et leur beauté due en grande partie à la présence de jardins hors de l'ordinaire et inspirés du Coran. Le secret des Almohades qui aménagèrent au $12^{\text {ème }}$ siècle les jardins de l'Agdâl et de la Ménara repose dans leur savoir-faire hydraulique. Ils inventèrent l'art des grands bassins, réservoirs. Océans artificiels, les réservoirs permettaient de disposer d'eau pour une mise en scène festive et l'irrigation de parcelles verdoyantes. Les Almohades ont ensuite propagé cette technique poussée de l'hydraulique jusqu'en Andalousie, permettant aux jardins de Grenade d'être à l'apogée de l'art des jardins.

Les structures «originelles» de ce type se développent sans modifications radicales jusqu'à la fin du $18^{\text {ème }}$ siècle. A partir de cette période, une rupture franche s'impose au tissu urbain des villes araboislamiques, un nouveau modèle absolu de ville se répand, qui remet en question les caractéristiques de la ville paradis islamique pour un nouvel idéal hygiéniste et ordonné rigoureusement. De nouvelles formes de jardins, les jardins publics, sont introduits, modifiant la perception et la structure de l'espace urbain et de la nature.

II) L'adoption de la modernisation « à l'occidentale » des villes arabes.

\section{1) La représentation occidentale de la ville devient le} canon de la modernité

En Europe, aux $18^{\text {ème }}$ et $19^{\text {ème }}$ siècles, s'affirme la volonté de réformer la ville. La rendre respirable du point de vue de l'hygiène et socialement, et volonté de pratiquer le loisir dans un cadre moral amènent à réfléchir sur les formes urbaines. La société de la fin du $18^{\text {ème }}$ et du $19^{\text {ème }}$ siècles projette son idéal social et sa représentation de l'espace sur la ville. La nature se situe au centre de ce projet car elle est parée de toutes les vertus. Présente partout 
sous la forme nouvelle de jardins publics, lieux de prestige social et urbain, elle est considérée comme ayant le pouvoir d'assainir la ville, de guérir la société et de la distraire tout en l'instruisant. C'est notamment dans cette perception de la ville, que sont réalisés les travaux d'Haussmann à Paris dans la seconde moitié du $19^{\text {ème }}$ siècle. Ses travaux ainsi que l'esprit dans lequel ils sont réalisés ont un impact très important sur la représentation des villes jusqu'en Orient.

L'Expédition d'Égypte (1798-1802), marque traditionnellement le début d'une influence occidentale importante sur le pays. A partir de cette période, l'influence française est, d'un point de vue politique, indirecte, mais très perceptible chez les dirigeants $d u$ pays et dans leurs modèles d'aménagement urbain. C'est le cas de Mohammed Ali, qui succède en 1805 aux Français à la tête de la province turque, secondé par des savants, des ingénieurs, des scientifiques, etc. qui restent en Égypte après le départ des troupes. Sous son gouvernement ont lieu les premières transformations (limitées) du Caire. Il introduit une nouvelle pensée de la ville (verte notamment), dont la réalisation symbolique la plus importante est l'aménagement de l'Azbakkiyya en parc public dès 1837.

C'est avec l'avènement d'Isma'îl, en 1863, que le Caire se transforme le plus. Les grands travaux du khédive prennent en partie leur source dans un séjour à Paris pour l'inauguration de l'exposition universelle de 1867, où les travaux d'Haussmann sont en voie d'achèvement. A son retour, Isma'îl se lance dans une vaste opération de modernisation du Caire, sur le modèle de Paris, pour offrir aux invités, à l'occasion de l'inauguration du Canal de Suez en 1869, une ville «moderne». Il s'entoure de conseilleurs et de techniciens occidentaux que lui recommande Haussmann. Un service des promenades et des plantations est créé par Barillet Deschamps (en 1870) sur le modèle de celui de Paris. La période de modernisation de la ville du Caire détermine un mode d'urbanisation qui, au fil des projets et réalisations, met à l'écart les tissus anciens traditionnels, en se référant toujours plus à un modèle européen de ville verte, absolument dominant, perçu comme le véhicule de la civilisation moderne.

Dans le même temps, la France poursuit une campagne d'expansion territoriale animée, au-delà des intérêts géopolitiques et économiques, par 
un credo colonial qui veut que la colonisation soit le symbole d'une certaine forme de civilisation. C'est dans ce contexte qu'au cours des quinze années qui suivent la première guerre mondiale, environ 120000 Français s'installent au Maroc considéré comme un pays neuf, un nouveau «Far Ouest », et que la population de Damas croît considérablement à partir de l'instauration du mandat français sur la Syrie en 1920. À Rabat comme à Damas, contrairement au Caire, l'influence française est donc directe et décalée d'environ un demi-siècle par rapport à l'Egypte. Au Maroc comme en Syrie, les Français prennent totalement en main la modernisation des villes. Ces dernières leur paraissaient chaotiques, désordonnées, sales, inextricables et labyrinthiques.

La modernisation urbaine, qui amène à la construction de nouvelles villes à côté des villes traditionnelles est un instrument d'affirmation d'une puissance étrangère. Les modèles urbains mis alors en place par l'administration française à Rabat et à Damas sont des plus modernes, propres à démontrer la suprématie culturelle et technique de la France et de légitimer sa domination coloniale. Plus encore, la construction des nouvelles villes est l'occasion d'inaugurer de nouveaux systèmes de construction. C'est ainsi que Lyautey à Rabat inaugure la planification dès les premiers projets de construction de Rabat (1913) alors que cette planification ne sera obligatoire en France qu'à partir de 1919 (loi Cornudet). Immédiatement appliqué à Damas, l'urbanisme de plan, assorti de "systèmes de parcs », permet de rationaliser la mise en place d'une ville moderne et verte selon les canons français. Le jardin devient un équipement urbain et un instrument d'éducation des populations à la morale, à l'ordre et à l'hygiène.

Au Caire, à Rabat et à Damas, les modernisations s'inspirent du seul modèle des villes rénovées en Europe à la même époque, qui deviennent l'étalon : planifiée, contrôlée, belle, claire, verte, alignée, saine et ouverte aux nouvelles pratiques du loisir. La ville arabo-islamique traditionnelle est dès lors définitivement déclassée, sa présence par le biais des médinas ou des quartiers anciens étant désormais perçue comme un relent d'archaïsme insupportable, face à des équipes de planificateurs qui veulent tendre vers l'avenir brillant promis par le modernisme, sous-tendu par l'hygiénisme. Les réalisations des experts et idéologues se traduisent ainsi concrètement 
dans la construction ex nihilo de nouvelles villes et de quartiers minutieusement planifiés, juxtaposés aux quartiers anciens, et par l'aménagement généralisé de jardins publics, qui eux aussi provoquent une rupture brutale.

\section{2) L'aménagement des jardins publics : la modification} des références urbaines et imaginaires

La modernisation des villes et l'adoption des canons européens entraînent l'abandon des références arabes dans l'architecture et l'aménagement, puisque ceux qui construisent ces nouvelles villes ne possèdent pas cet arrière plan culturel (ils sont Français, comme à Rabat et à Damas) ou souhaitent s'en défaire (comme au Caire). On assiste par conséquent à une rupture fondamentale et brutale dans la conception de la ville, ainsi que dans celle de la nature en ville. De nombreux jardins publics, permettant l'ostentation d'une politique urbaine moderne, sont créés dans les villes.

Le jardin de l'Azbakkiyya aménagé au Caire par Mohammed Ali, et le quartier qui l'entoure étaient devenus depuis les années 1830 le centre de la ville moderne et le point de rencontre avec les quartiers plus « traditionnels ». Sous Isma'îl qui réaménage le quartier à partir de 1868, le jardin est ramené à un quart de sa surface initiale et prend des allures de parc parisien. Sa composition paysagère est en effet réalisée par Barillet Deschamps qui, dit-on, se serait inspiré du Parc Monceau de Paris dont il était l'auteur. 


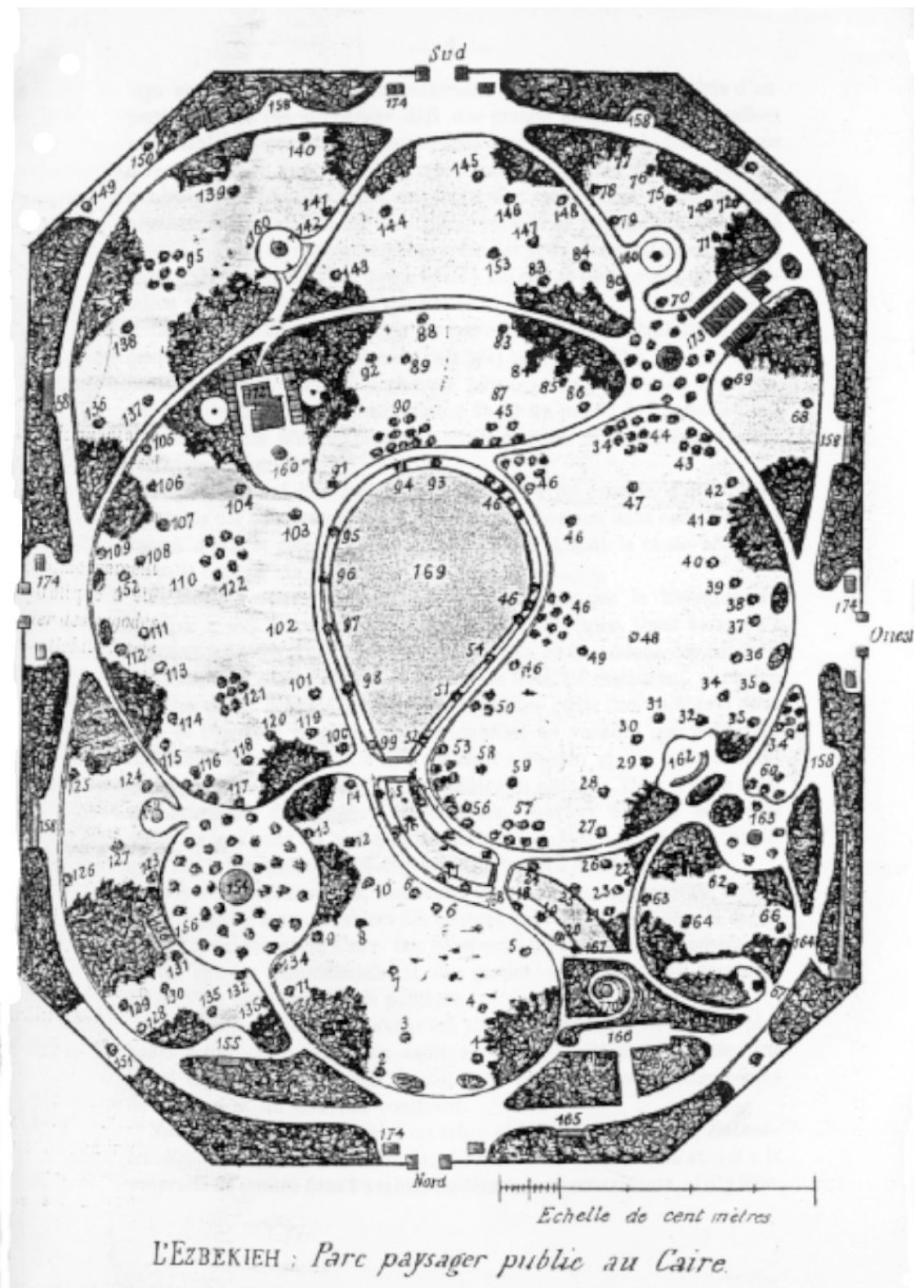

Plan du jardin de l'Azbakiyya, (Le Caire). On observe le plan des allées en « serpentine » d'inspiration « anglo-chinoise », le lac central $\left(\mathrm{n}^{\circ}{ }^{159}\right)$, les kiosques à musique (154 et 173) et la grotte $\left(n^{\circ} 166\right)$.

(Delchevalerie, Le parc public de l'Ezbekieh au Caire, Gand, impr \& lithogr C. Annoot-Braeckman, Ad. Hoste, succr, 1897, 16 p.).

Les règles européennes pour l'aménagement des jardins sont dominantes: on n'y trouve aucune référence à l'art des jardins araboislamiques. Il n'y a pas dans ces jardins de fontaines aux mosaïques, mais des petits lacs destinés parfois au canotage, la géométrie rectangulaire et divisée en quatre rectangles du jardin arabo-islamique est complètement supplantée par la géométrie à la française et/ou les allées sinusoïdales et 
romantiques du parc « anglo-chinois » à la mode en cette seconde moitié du $19^{\text {ème }}$ siècle en Europe. Sont ainsi également créés au Caire sur le même modèle, le jardin zoologique et botanique (1861/1871), conformément à ceux qui sont à la mode en Europe et qui « excitent à juste titre l'admiration universelle $»^{14}$, ainsi que l'Aquarium en 1874. Grand parc public, ce dernier occupe une surface importante de l'île de Gezira, dont les Anglais font un club à partir de 1884, n'en laissant qu'une petite partie pour le jardin. Un jardin japonais planté de bambous et décoré de statues de Bouddha et de lanternes japonaises est même créé à Helwan ${ }^{15}$.

A Rabat, la période coloniale a été propice au développement des grandes infrastructures et on peut la distinguer pour ses réalisations en matière de jardins publics. Prévus dans le plan d'aménagement de Prost, les jardins sont réalisés assez rapidement, grâce à la création du service administratif des plantations et promenades de Rabat en 1921, sur le modèle du service du même nom à Paris. Le jardin du Triangle de Vue, d'une surface de 7 ha est situé en face de la médina. Il est au centre de la ville nouvelle de Rabat. Dessiné autour d'un axe central d'où partent des contreallées dans un style plutôt «à la française », il est largement boisé et clôturé d'une grille monumentale. Le bois de l'Agdal, situé en périphérie, au sudest de la ville, fait également partie des réalisations de la période Prost à Rabat. Clairement inspiré des bois de la périphérie de Paris, le bois de l'Agdal marquait le prestige de la capitale du protectorat français. Les jardins du Belvédère, du Chellah, le jardin d'Essais et le jardin de la casbah des Oudaïas complétaient le système de parcs de Rabat. S'ils s'inspiraient majoritairement de l'art des jardins en vogue en France, et le revendiquaient ouvertement, les paysagistes de Rabat étaient pourtant davantage influencés par l'art des jardins arabo-islamiques que ne l'avaient été les concepteurs des jardins publics du Caire.

A Damas la nécessité de créer des jardins s'imposait avec moins de force qu'au Caire ou à Rabat, puisque la ville était entourée d'une oasis verdoyante, fertile, riche et accessible. De plus, la ville possédait déjà

\footnotetext{
${ }^{14}$ « Note sur les résultats obtenus au jardin d'acclimatation du Caire pendant la période de deux années » du Professeur Gastinel, membre de l'Institut Egyptien, Bulletin de la Société Impériale d'Acclimatation, Paris, 1865.

${ }^{15}$ Ville industrielle nouvelle située au sud du Caire, et aujourd'hui agglomérée à la métropole.
} 
quelques jardins, aménagés à l'époque de modernisation ottomane, notamment sur la route de Beyrouth. Le plan d'aménagement adopté en 1937 prévoyait néanmoins quelques espaces de verdure, d'une surface réduite, mais qui affirmaient les références françaises de la conception d'une ville. Le jardin public situé entre le lycée français et la route de Beyrouth est réalisé dès 1936, avant l'adoption du plan d'aménagement, puis le jardin AlMunchiyeh sur la rive droite du Barada, en face de la Tekkiyeh Solimaniyeh est aménagé selon des plans très simples et très classiques de jardin public sans panache en France (des gazons, des parterres, des allées géométriques « à la française » et contre-allées sinusoïdales, des bancs et des arbres), ainsi que le jardin Zénobie, plus au nord et le petit jardin des Canons. Réalisant davantage des squares que des parcs publics, les aménageurs de Damas tels que René Danger s'appuyaient sur la prégnance de l'oasis, qu'ils considéraient comme le parc public principal de Damas. Dans tous les cas, les références à la fois dans le tracé et les plantations étaient profondément françaises.

L'introduction de jardins publics inspirés de la France dans les villes du Caire, de Rabat et de Damas, a été perçue par la population locale comme un bouleversement des références, des comportements, et du paysage. Il était désormais possible de s'installer pour un temps très court dans un environnement de verdure. La nature, sous forme de végétation organisée, devenait publique et visible. Dotée de bancs, de grosses fontaines ostentatoires, de parterres, elle s'offrait à tous. Elle avait aussi changé de nature par rapport aux jardins arabo-islamiques des grandes demeures. Khaled Ziadé dans son roman Vendredi Dimanche décrit les transformations de Tripoli (Liban) lors du mandat français, que l'on peut considérer comme applicables à Damas à la même époque, ainsi qu'en terme de rupture, au Caire et à Rabat.

«On inaugura le jardin public, planté de fleurs d'ornement qui ne dégageaient aucun parfum et que l'on avait importées de l'étranger. Au milieu il y avait un bassin avec une fontaine que nous ne nous lassions pas, enfants, de contempler. C'était un jardin géométrique, avec des parterres symétriques composés de plantes sans odeur et non 
pas de rosiers, d'œillets et de jasmin, toutes ces fleurs qui poussaient au hasard entre les vieilles maisons. L'ensemble était entouré d'une haie d'arbres inconnus, des buis qui avaient un feuillage très vert et ne donnaient pas de fruits ! ».

Ainsi, la nature introduite en ville et offerte aux habitants était d'un caractère très différent de celle que l'on trouvait jusque là dans les jardins des cours intérieures ou dans les vergers autour des villes où l'on rencontrait alors couramment des orangers, des citronniers, des figuiers, lauriers, bananiers, mûriers, poiriers ou abricotiers. On y cultivait des fleurs sans aucun ordre, des aromates (menthe, basilic...), des plantes grimpantes comme le jasmin, le chèvrefeuille ou le rosier, le tout dans une forme de désordre apparent, expliqué par Jean Gallotti par le fait que «dans ces climats trop secs, ils représentent, non pas l'hostilité de la nature, mais son sourire » et par conséquent, « les arbres croissent ignorants des cisailles $»{ }^{16}$, contrairement aux jardins à la française où l'homme exerce son empire sur la nature en la taillant, la domestiquant. La rupture dans le paysage et la représentation traditionnelle et idéalisée de la nature en paradis terrestre était désormais consommée. Et pourtant, à certains endroits, des références à la ville arabo-islamique ont été introduites dans la ville moderne. On les retrouve dans la conception de quelques jardins publics.

\section{3) Au sein de cette révolution urbaine, une résistance esthétique : des jardins publics orientalisants}

Les Français qui arrivent au Caire avec l'Expédition d'Egypte en 1798, à Rabat en 1911 et à Damas en 1920, ne trouvent pas l'Orient auquel ils songeaient. On observe alors chez eux une forme de désenchantement. À défaut d'accéder au rêve oriental, certains d'entre eux vont chercher à le créer. Profitant de l'importante vague de construction, des jardins « arabes » sont aménagés au Caire et à Rabat, mettant explicitement en avant les références mythiques des jardins d'Andalousie. C'est ainsi que sont créés le

\footnotetext{
${ }^{16}$ Le jardin et la maison arabes au Maroc, p. 16
} 
jardin al-Andaluz, près du Nil au Caire et, à Rabat, le jardin de la kasbah des Oudaïas et le jardin d'Essais.

Dans la vague de création de jardins publics «à la française » ou «à l'anglo-chinoise » au Caire, le petit jardin Al-Andaluz fait exception. Aménagé dans les années 1870 sur l'île de Gezira, il est dessiné selon le plan géométrique du chahar bagh, ses allées sont dallées de mosaïques et une fontaine à vasque emplit l'air d'un murmure hydraulique. Les essences plantées sont des roses, des jasmins, des lauriers et grâce aux haies épaisses qui l'isolent du reste de la ville, on pourrait s'imaginer au centre d'une maison à cour intérieure. Seul exemple de l'influence de l'art araboislamique des jardins sur les jardins publics au Caire, Al-Andaluz est sans doute un des jardins les plus connus du Caire, alors qu'il est aujourd'hui fermé au public.

A Rabat, Forestier souhaite tenir compte de la culture « locale » dans ses réalisations marocaines. Il conçoit en 1914 un jardin public de style andalou, réalisé en 1919, dans la cour du musée de la Casbah. Dessiné selon un plan orthogonal le jardin respecte les éléments du riyâd marocain décrit notamment par Jean Gallotti ${ }^{17}$. L'espace semblait convenir parfaitement à l'aménagement d'un tel jardin puisqu'il était clos par les hautes murailles de la citadelle de Rabat, et était de forme rectangulaire, ainsi que doivent l'être les riyâds. Une allée axiale croise plusieurs allées transversales en angle droit, reproduisant ainsi le plan géométrique du chahar bagh. Les parterres sont situés dans les espaces qui séparent toutes ces allées et sont franchement en contrebas de ces dernières, afin que les visiteurs ne marchent jamais sur la terre, et que les parterres profitent au maximum de l'eau de pluie ou d'irrigation. Acquis au mythe andalou du califat de Cordoue, Forestier réinvente la tradition du jardin arabo-islamique dans la Casbah des Oudaïas. Il la réinterprète en utilisant des références qu'il veut locales (alors qu'on n'en avait pas d'exemple à Rabat) et un savoir faire technique occidental.

\footnotetext{
${ }^{17}$ Le jardin et la maison arabes au Maroc, p. 14
} 


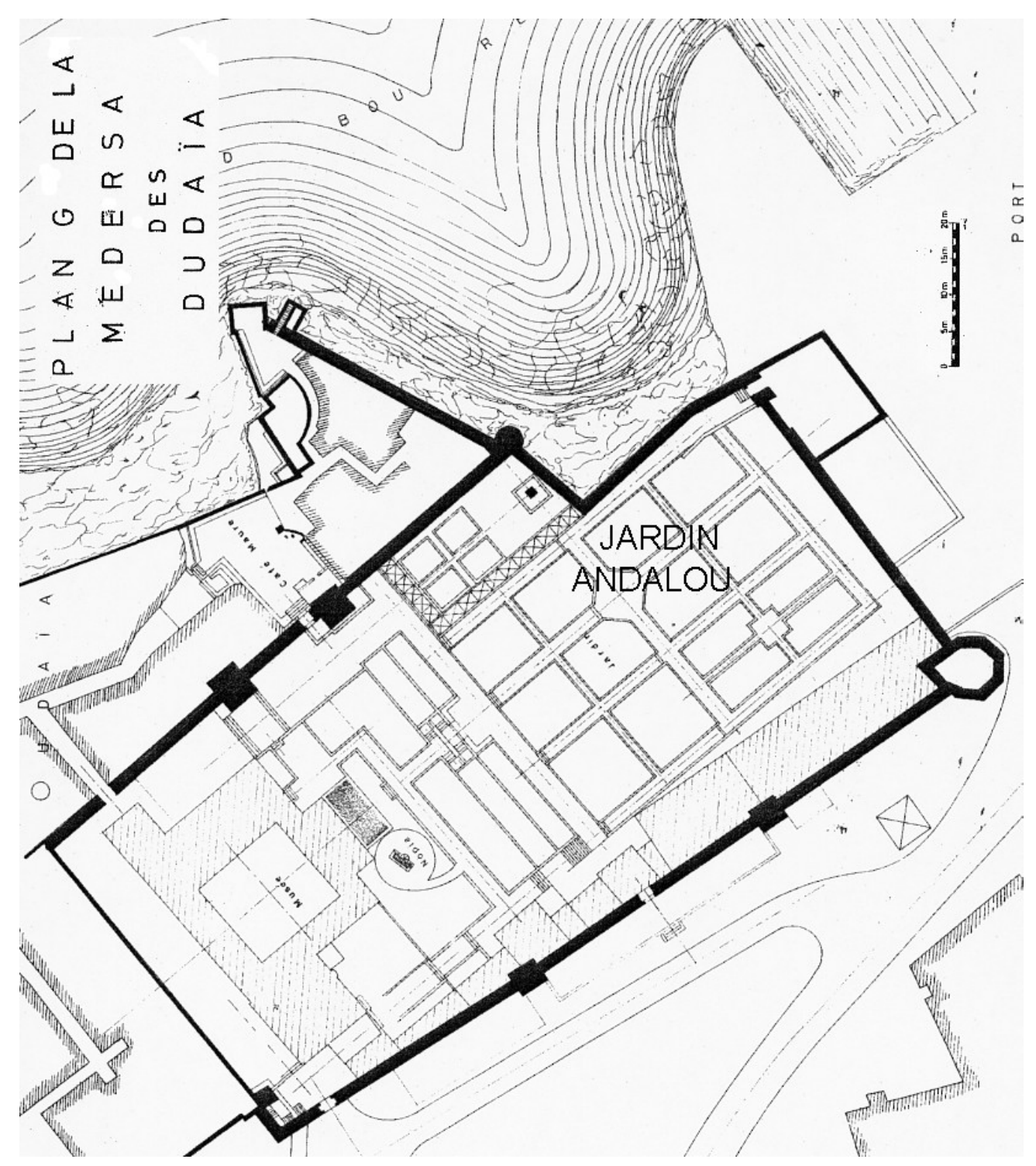

Plan de la medersa des Oudaïas, Rabat.

On observe le plan d'inspiration chahar Bagh du jardin, dessiné par Leforestier.

Ce plan adopte exactement le modèle perse et arabo-musulman du jardin traditionnel. (source: Ministère des Affaires Culturelles, Direction du Patrimoine, Rabat, 1994).

Le jardin d'Essais qu'il réalise également à l'Agdal de 1919 à 1922 fait moins référence à l'art des jardins arabo-islamiques. Il est davantage un essai de synthèse entre l'Orient et l'Occident. Forestier reprend la structuration en terrasses devenue mythique depuis les jardins de Babylone dans l'imaginaire jardinier de l'Orient, et profite de la pente pour faciliter l'irrigation. Mais là s'arrêtent les références orientales car les allées et les contre-allées droites, perpendiculaires rappellent la structuration d'un jardin classique à la française. 
Ainsi, si le jardin des Oudaïas et le jardin Al-Andaluz sont inspirés du jardin arabo-andalou, leur conception et leur réalisation sont entièrement assumées par des Français, qui réalisent leur rêve d'Orient à cette occasion.

Malgré ce petit îlot de résistance esthétique, la modernisation des villes du monde arabe a été synonyme de l'abandon radical des traits communs de la ville arabo-islamique, et de l'adoption d'un modèle occidental devenu totalement dominant. De nombreux jardins publics ont été réalisés pendant cette période de rupture et ont constitué des lieux de prestige politique, d'affirmation de la puissance conquérante et de la domination d'une culture. Ils ne le resteront pas. Confrontées à une croissance incontrôlée, les villes les abandonnent, puis, dans une volonté de reprise en main de l'espace urbain, les privatisent peu à peu. Dès lors, ces jardins se transforment en espaces commerciaux qui donnent aussi naissance à de nouvelles formes de lieux de loisirs, les parcs d'attractions.

\section{III) De l'abandon à la privatisation : les jardins contemporains, et les parcs de loisirs}

\section{1) Le jardin public perd sa symbolique de modernité face à une croissance urbaine incontrôlée}

La période des indépendances (globalement les années 1950) est marquée par une croissance urbaine très rapide que les nouveaux Etats ont du mal à gérer. Parallèlement les représentations de la ville évoluent encore et l'idée s'affirme que l'on peut contrôler l'expansion urbaine en appliquant des règles techniques basées sur des calculs rationnels. Des solutions «miracles » sont inventées et se répandent partout, telles que les ceintures vertes (très en vogue dans les années 1970-1980), et l'adoption de quotas d'espaces verts. La nature a en effet changé de représentation et on la considère selon un aspect fonctionnel, depuis l'adoption des principes de la Charte d'Athènes. Les solutions proposées, face à la croissance 
exponentielle du Caire, de Rabat et de Damas sont issues d'urbanistes français. L'étalon de la modernité urbaine à cette époque vient toujours de l'Occident.

Ainsi, à Rabat, c'est Michel Ecochard qui imagine un nouveau plan d'aménagement dès 1947 qui n'est remplacé qu'en 1972 par un premier schéma directeur d'aménagement et d'urbanisme, disposition simplement transposée de la France, dont le but était d'organiser, de structurer et d'orienter la croissance de la capitale marocaine. Au Caire aussi un premier schéma directeur est adopté, en 1953, qui tente de mettre en place une politique volontariste dont le principal but est de décongestionner la ville. A Damas, c'est encore Michel Ecochard, associé à l'urbaniste japonais Banshoya, qui est chargé d'élaborer le nouveau schéma d'aménagement en 1968 afin de remplacer celui de 1936 désormais insuffisant. Ces trois villes ayant connu une modernisation selon un modèle similaire sont confrontées à des problèmes semblables : l'extension « en tache d'huile» de la ville, la multiplication des zones d'habitat spontané, l'insuffisance des équipements collectifs et l'engorgement des voies de circulation.

A ces problèmes que rencontrent la plupart des villes, les villes du monde arabe n'inventent pas de réponse propre: ils font appel à des spécialistes de la gestion urbaine et aux techniques nouvelles d'urbanisme. Toujours à la recherche de l'affirmation de la modernité de leurs cités, et nostalgiques de la période de modernisation, les pays cherchent à appliquer des normes internationales dont ils attendent une reconnaissance de leur appartenance au « monde civilisé ». Ainsi, après une période d'abandon des jardins publics, les municipalités découvrent l'espace vert dont l'OMS recommande qu'il atteigne $10 \mathrm{~m}^{2}$ par personne.

La notion de ville verte développée par les hygiénistes du $19^{\text {ème }}$ siècle, redevient l'idéologie dominante, et se réinvente comme une idée nouvelle. Elle est alors sous-tendue par les questions d'écologie, d'environnement, de qualité de vie et, dans une «sorte de compulsion, une motivation époquale irraisonnée, [s'impose] au XXème siècle l'idée que la ville ne peut être belle et bonne à vivre qu'au-delà d'une certaine proportion d'espaces verts $»^{18}$.

\footnotetext{
${ }^{18}$ Augustin Berque, « Jardins et urbanité : regards croisés Europe-Asie », dans Mercier et Bethemont, La ville en quête de nature, p. 39.
} 
Les schémas directeurs prévoient donc à Rabat, au Caire et à Damas l'aménagement d'espaces verts, qui ne sont pas des jardins. Ces derniers ont en effet peu à peu perdu, depuis la fin des années 1950, leur prestige et leur rôle d'affirmation de la puissance publique qui se sont davantage incarnés dans l'architecture, reléguant les jardins publics au rôle d'écrin des bâtiments prestigieux. «Les surfaces vertes (...) n'auront pas pour unique fonction l'embellissement de la cité. Elles devront, avant tout, jouer un rôle utile $\gg{ }^{19}$ ainsi que l'énonce la Charte d'Athènes. L'embellissement ne passe plus par la qualité de la verdure présente dans la ville, mais par son organisation rationnelle, fonctionnelle et surtout par sa quantité. Surfaces vertes ou espaces verts sont des notions récentes ${ }^{20}$, beaucoup plus larges que celle de jardin public, d'origine occidentale et qui se sont imposées avec force dans tous les aménagements concernant Le Caire, Rabat et Damas, exportés avec l'urbanisme.

La mutation du vocabulaire est peut-être à l'origine (ou est la manifestation) d'un changement de perception de ces types d'espaces. Si au $19^{\text {ème }}$ et au début du $20^{\text {ème }}$ siècle les jardins publics étaient parés de vertus sociales, morales et esthétiques, et étaient donc conçus par des spécialistes de l'art du jardin, ils se dissolvent dans le dernier tiers du $20^{\text {ème }}$ siècle dans une catégorie lâche d'équipements qui traduisent un esprit comptable de la ville, où tout est question de proportion. Leur conception est désormais confiée à des ingénieurs plus qu'à des paysagistes. Difficile dans ce cadre de les transformer en espaces d'identité culturelle spécifique.

Le recours au Caire, à Rabat et à Damas aux mesures techniques occidentales affirme avec force que les villes du mondes arabe ne cherchent pas, ou ne sont pas en mesure de chercher, des solutions qui affirmeraient une spécificité de leurs villes comparativement aux villes occidentales. Au contraire, plus que jamais leurs modèles urbains se situent très ouvertement dans les pays développés : en Europe (Paris, Londres), en Amérique du

\footnotetext{
${ }^{19}$ La Charte d'Athènes, p. 61-62

${ }^{20}$ Ces notions sont liées à la transformation des éléments de la ville en «équipements 》. Les espaces verts doivent être répartis équitablement dans les divers quartiers de la ville. Ceci entraîne par conséquent une planification par ratio. La notion d'espace vert a été utilisée pour la première fois en France n 1903 par l'urbaniste Hénard. Mais elle n'entre officiellement dans les textes qu'en 1961. Ces textes sont ceux sur lesquels la notion d'espace vert est bâtie à Rabat (1978) et à Damas (1968, avec le plan de Banshoya et Ecochard), bien que utilisée avant même leur reconnaissance juridique.
} 
Nord (New York) et même au Japon (Tokyo). Ainsi les solutions importées sont vues comme les meilleures.

Avec la fonctionnalisation des espaces dans les villes, la proportion et la gestion comptable, les jardins publics comptés parmi les espaces verts deviennent des espaces comme les ronds points paysagés ou les entrées de villes fleuries. Ils ne sont plus guère différents des autres espaces ouverts des villes. Ainsi, dépossédés de leur rôle social et civique, les espaces publics, et notamment les jardins publics, évoluent. Une partie des habitants se retire de l'espace public, le laissant aux couches populaires. Désormais le pouvoir ne s'y met plus en scène, et l'entretien est jugé trop coûteux. Il peut donc être privatisé et laissé à l'initiative privée. Par ailleurs, les sociétés ont évolué et le loisir populaire qui se développe, surtout depuis les années 1980, a suscité une demande en équipements de distraction, entraînant une privatisation des espaces publics de loisirs dont l'évolution s'est rapidement faite sur le modèle des parcs d'attractions, devenant les seuls modèles de référence.

\section{2) Concessions et transformation des jardins en espaces commerciaux}

Le Caire, Rabat et Damas ont toutes trois connu l'influence de la Charte d'Athènes dans leurs schémas d'aménagement et d'urbanisme. Or celle-ci considère que les espaces de loisirs sont un droit auquel la ville doit subvenir et répondre à travers des espaces suffisants et adéquats. Or, les pouvoirs publics restent défaillants pour fournir des équipements et des structures de loisirs suffisants. Ceci amène les collectivités locales à chercher des substituts et des « arrangements économiques » à leur mission d'aménagement, qui peuvent être des associations d'habitants, mais également, pour les espaces de loisirs, des sociétés privées à qui les pouvoirs représentatifs de l'Etat délèguent leurs pouvoir et confient, sous forme de concession, des pans entiers de l'espace public ${ }^{21}$.

\footnotetext{
${ }^{21}$ Voir Signoles, et alii, L'urbain dans le monde arabe
} 
Selon cette logique, les jardins publics se trouvent découpés en des lieux dont la fonction est différente, à la faveur de concessions à des sociétés privées. La plupart des espaces concédés dans les jardins publics sont équipés de manèges et leur accès devient payant. Clos, surveillés, les Luna Parks des jardins publics, retirent à l'usage public et gratuit une partie de la surface du jardin. Les équipements de fête introduisent une discrimination d'accès fondée sur les revenus. On les trouve désormais partout. Les jeux qui varient du simple toboggan aux manèges pour adultes sont tous payants individuellement. Ils rencontrent cependant un vif succès. La pratique des manèges n'est pourtant pas une nouveauté puisqu'ils sont une tradition très ancrée dans les habitudes de fête, notamment des Syriens et des Egyptiens. Associés aux fêtes des Saints musulmans au Caire (mouled-s) et à toute fête religieuse en Syrie, ils sont depuis l'époque ottomane installés dans les rues à ces occasions. Mais désormais, ce qui est recherché est le manège à sensations, sur le modèle de ceux que l'on trouve dans les fêtes foraines en Europe, et plus encore, sur le modèle des parcs d'attractions d'inspiration nord-américaine.

Ainsi, devant la forte demande de ce type d'équipements, et l'aubaine financière que cela représente (les propriétaires des manèges payent un loyer au jardin et assurent l'entretien de leurs machines), les services municipaux n'ont pas hésité à les introduire dans les jardins publics. Un second type de concession privée dans les jardins, l'implantation de parcs d'attractions à part entière, est encore davantage représentatif de l'évolution des jardins publics vers un modèle qui s'internationalise. Ceci est surtout vrai au Caire où l'on peut prendre l'exemple du Jardin International. Wonder Land a été créé près de dix ans après l'inauguration officielle du jardin en mars 1987. Sa référence explicite à «Disney Land» ainsi que les « services » qui y sont associés (un Mac Donald's, six salles de cinéma à grand écran, notamment) sont utilisés pour attirer des visiteurs, mais servent également à produire une image « moderne » du jardin, devenu dès lors un simple décor.

A la suite du succès de telles implantations, se sont développés dans la proche périphérie du Caire, sur ses marges désertiques, des parcs d'attractions totalement privés. En Égypte leur développement est évident 
depuis les années 1990. On trouve Aquapark, à l'ouest du Caire sur la route de Suez, et plusieurs nouveaux parcs près de la ville satellite du Six Octobre, à l'est du Caire en direction du Fayoum, dont Dream Park. Leur référence à la culture américaine se lit notamment dans leurs noms à consonance anglo-saxonne. Les visiteurs s'acquittent d'une entrée forfaitaire qui leur donne un droit d'usage pour un certain nombre de manèges.

Ces parcs de loisirs n'ont de parcs que le nom «parc» (devenu « bark » en arabe) car ils ne sont pas en réalité des espaces de « nature », le végétal étant une forme de décor et la priorité donnée aux équipements de fête. Ils sont la manifestation d'une nouvelle conception du loisir, de la société, et de la nature. Au milieu des lumières multicolores, de la musique anglo-saxonne, de la foule, de la fête, des ballons Mac Donald's et des milk shakes, le bonheur paraît à portée de main, un bonheur prêt à consommer et international. Les références à la culture locale sont très peu présentes. A Dream Park, si l'on trouve parfois la statue colorée d'un sphinx à l'entrée d'une grande roue, l'ambiance qui domine le décor est le far ouest et le monde enchanté et pastel des dessins animés, qui coexistent dans un même espace.

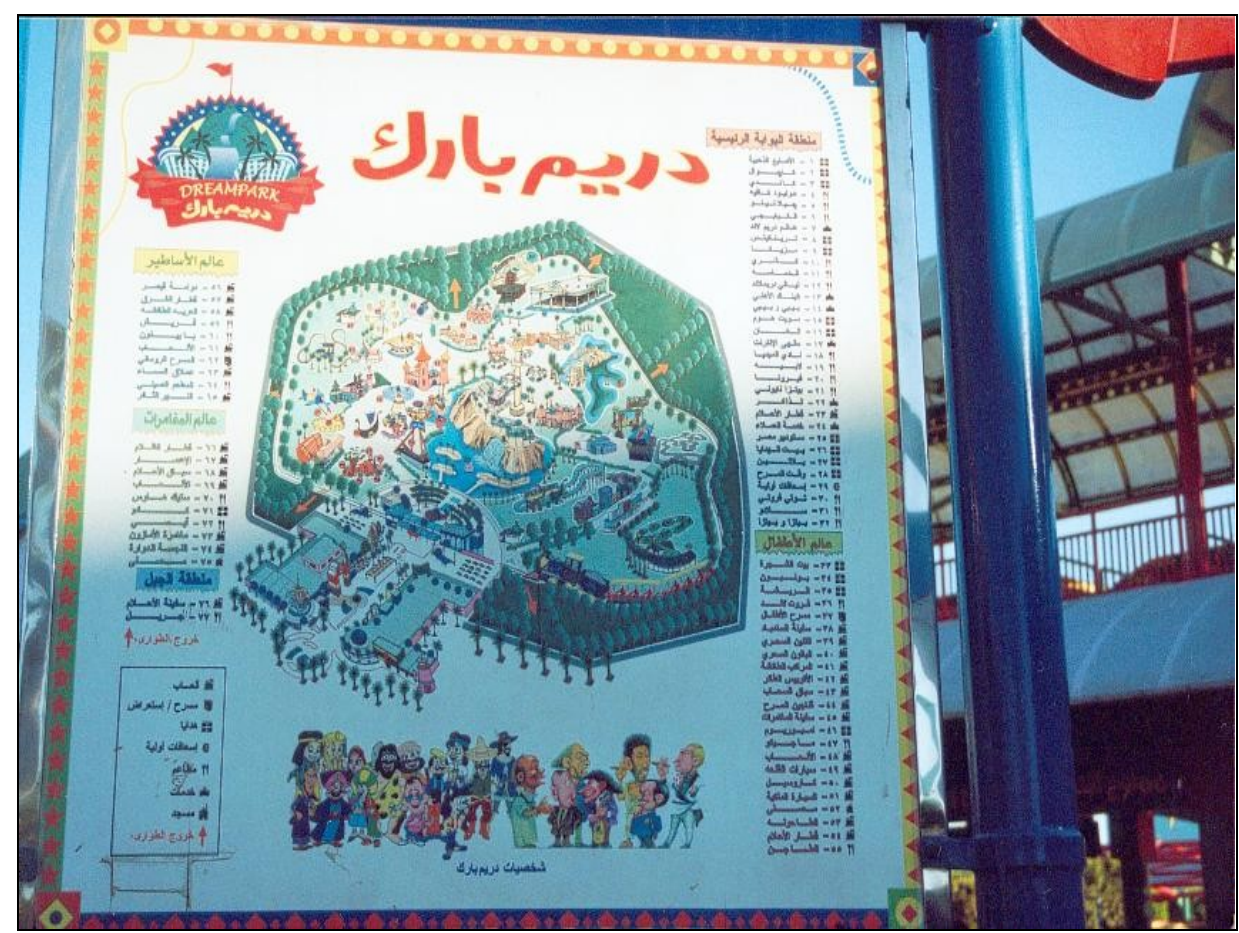

Plan de Dream Parc affiché à l'entrée du parc d'attractions). La nature n'est qu'un écrin aux nombreux manèges, dans un espace clos. (Le Caire, juin 2004) Photo G. Gillot 


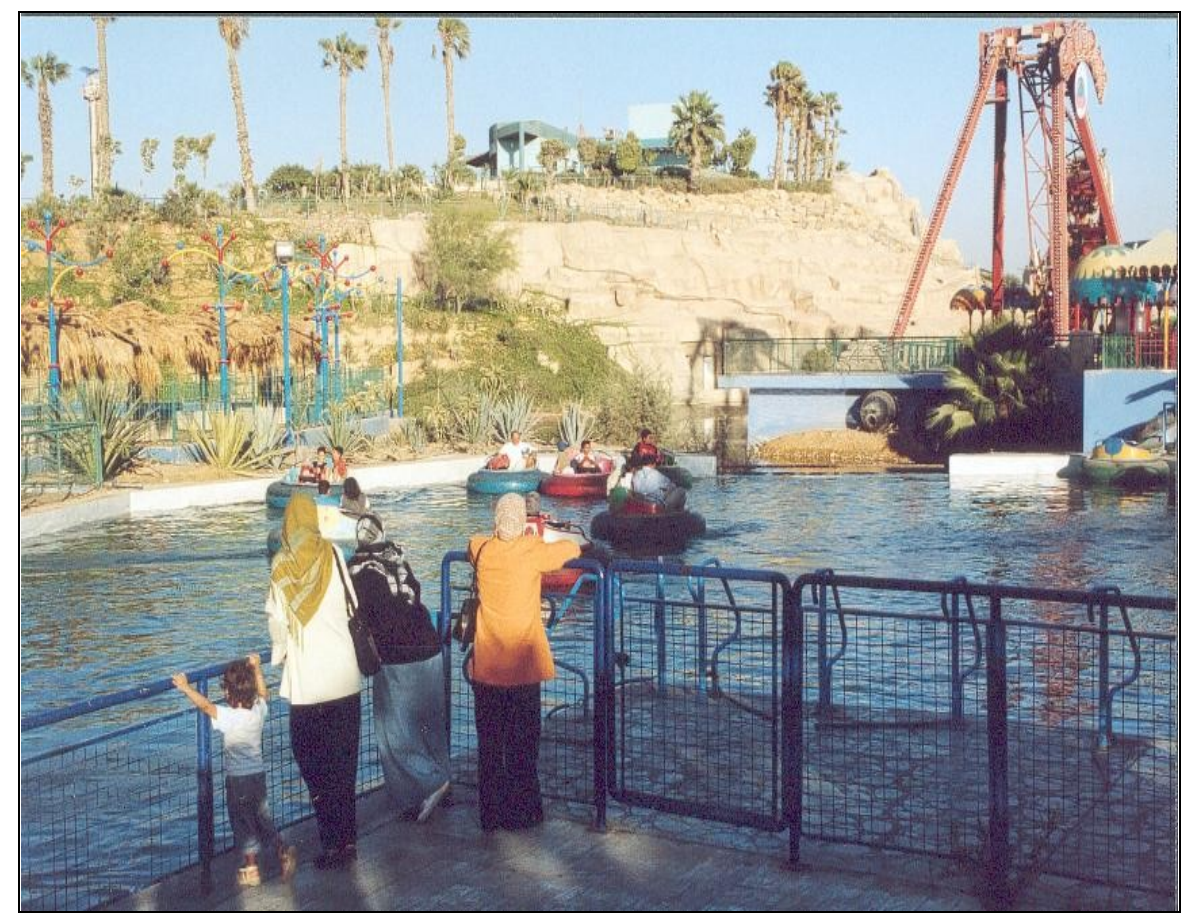

A Dream Park (Le Caire), le rêve d'une eau abondante et ludique en plein désert. (juin 2004) photo G. Gillot

Comme évacuées de ces lieux d'euphorie, sortes d'îlots sécessionnistes, les références vernaculaires n'apparaissent quasiment nulle part. Peut-être est-ce parce que ces parcs représentent un univers de fiction et par conséquent ne peuvent/doivent pas être spatialisés, de la même façon que le paradis. Ils le sont pourtant par leurs références à l'Amérique. Mais l'Amérique, par l'exportation importante de ses restaurants rapides et parcs de loisirs est désormais présente dans le monde entier, représentant sans doute un modèle perçu davantage comme international qu'étasunien. Mondes clos et séparés du reste du territoire par des frontières franches, accessibles sous conditions, comme l'étaient dans la culture arabo-musulmane les jardins d'agrément privés, ils attirent par la promesse d'un plaisir immédiat, d'une rupture avec le quotidien et l'illusion de la facilité de la vie dans un environnement où tout est étudié pour provoquer une forme d'enchantement. Mais là où l'on devait entretenir des liens sociaux pour y avoir accès, ou le posséder soi-même, il suffit désormais de payer une entrée pour en profiter. 


\section{CONCLUSION}

Un siècle après l'introduction en partie brutale des nouvelles formes urbaines, dont les jardins publics, par la transposition des canons modernes des villes occidentales aux villes du monde arabe, et une forme de mondialisation des modèles par la colonisation, le mythe des jardins araboandalous est toujours vivace. Il est pourtant peu représenté sur un terrain où la spécificité arabo-islamique des villes, considérée d'abord comme archaïque, a longtemps et autant que possible été gommée de la ville moderne, et où, témoins de l'importante référence à l'Occident, les nouveaux lieux de loisirs de plein air sont sur le modèle des parcs d'attractions américains. Cependant, le jardin de la kasbah des Oudaïas, ainsi que le Jardin d'Essais à Rabat sont classés sur la liste du patrimoine national marocain. Ils sont considérés comme représentatifs d'un art local et objets d'une revendication identitaire ${ }^{22}$. Par la reconnaissance récente d'un tissu urbain dans son entier (les médinas au Maghreb, la ville islamique au Caire) comme appartenant au patrimoine de l'humanité, les caractéristiques de la ville arabo-islamique ont été considérées comme des spécificités à protéger afin qu'elles ne disparaissent pas. Cette redécouverte d'un patrimoine urbain hier dénigré et abandonné semble faire l'objet, à l'occasion de sa rénovation, d'une mise en scène dans une forme de réenchantement des villes araboislamiques selon les représentations, toujours en cours, de la «ville orientale » telle que la rêvaient les voyageurs dans les pays du Levant au $19^{\text {ème }}$ siècle. Elle marque peut-être aussi une forme de réappropiation de l'âge d'or de l'identité arabo-musulmane dans ce qu'il a de plus raffiné, un moyen peut-être pour les pays arabes de se distancier de l'image de pays en développement et stigmatisés dans les relations internationales.

La mise en valeur du patrimoine semble être devenue l'occasion de fabriquer un rêve oriental presque totalement absent des villes modernisées, qui correspondrait à un regard occidental, touristique. Exacerbée dans une théâtralisation sensée représenter l'ensemble de ses caractéristiques, la ville arabo-islamique redécouvre des spécificités et les caricatures pour induire un dépaysement. Ainsi, telle un parc d'attractions architectural arabo-musulman,

\footnotetext{
${ }^{22}$ Voir Gillot, Gaëlle, « La nature urbaine patrimonialisée... »
} 
on a construit à Tunis une nouvelle médina « traditionnelle » sur mesure pour les étrangers ${ }^{23}$. Le retour récent à l'aménagement ou à la rénovation de jardins sur le modèle arabo-andalou, et l'abandon des jardins publics à des sociétés privées qui les suréquipent, traduisent là encore une modification de l'esprit de la ville. L'étude de l'évolution de la forme des jardins ainsi nous éclaire sur les fonctions projetées sur la ville. Pour continuer à attirer des touristes (nationaux et internationaux), grosse source de devises, les villes du monde arabe doivent s'adapter à l'évolution d'un commerce en mutation, et proposer à leurs visiteurs un monde différent, en quelque sorte enchanté, qui corresponde aux attentes et aux promesses de l'Orient vantées dans les magazines des agences de voyage. La redécouverte des spécificités débouche-t-elle alors forcément sur leur artificialisation ?

\section{BIBLIOGRAPHIE INDICATIVE}

Arnaud, Jean-Luc, Le Caire. Le Caire. Mise en place d'une ville moderne. 1867-1907, Arles, Sindbad, Actes-Sud, 1998

Auffret, Séverine, Aspects du Paradis, Paris, Arléa, 2001

Barcelo Miquel, Gillot Gaëlle, Marquez Maite, Pelat Frédéric, Piera Arcadi, Les jardins de la vieille ville de Sanaa, Centre Français d'Archéologie et de Sciences Sociales de Sanaa, Universitat Autonoma de Barcelona, Bellaterra, mars 2004

Baridon, Michel, Les jardins. Paysagistes-Jardiniers-Poètes, Paris, Robert Laffont, 1999

Brookes, John, Gardens of paradise. The history and design of the great Islamic gardens, London, Weidenfeld and Nicholson, 1987

Collectif, Forestier, Jean-Claude-Nicolas. Du jardin au paysage urbain, Paris, Picard, 1990

Gallotti, Jean, La maison et le jardin arabes au Maroc, Paris, Lévy, 1926

Gardet, Louis, La géographie du monde musulman, Paris, La Haye, NewYork, Mouton (éditions de l'EHESS), 1980

Gastinel, «Note sur les résultats obtenus au jardin d'acclimatation du Caire pendant la période de deux années », Bulletin de la Société Impériale d'Acclimatation, Paris, 1865.

\footnotetext{
${ }^{23}$ Voir à ce propos la communication de Pierre-Arnaud Barthel « Enchanter les touristes en médina : mises en scène et construction de lieux « orientalisants ». Les cas de Tunis et de Yasmine Hammamet (Tunisie)», atelier « Enchantement, réenchantement des territoires ?» (sous la direction de F. Bruyas et G. Gillot), Afemam 2004, disponible en pdf sur le site internet « réenchantement » (voir bibliographie).
} 
Gillot, Gaëlle, «La nature urbaine patrimonialisée : usage et perception. Le cas de deux jardins marocains », dans Habiter le patrimoine, Maria GravariBarbas (dir.), (CD-Rom, nov. 2004, et ouvrage aux PUR en cours)

Gillot, Gaëlle, Ces autres espaces. Les jardins dans les grandes villes du monde arabe. Politiques et pratiques au Caire, à Rabat et à Damas, thèse de doctorat de géographie, sous la direction de J-F Troin et J-C Depaule, Université de Tours, 2002

Le Corbusier, La charte d'Athènes, (1957), Paris, Points, 1994

Mercier Guy, Bethemont Jacques, (dir.), La ville en quête de nature, Québec, Septentrion, Centre Jacques Cartier, 1998

Mosser Monique, Nys Philippe (dir.), Le jardin, art et lieu de mémoire, Besançon, Les Editions de l'Imprimeur, 1995

Nicolet Claude, Ilbert Robert, Depaule Jean-Charles, (dir.), Mégalopoles méditerranéennes. Géographie urbaine rétrospective, Paris, Maisonneuve \& Larose, MMSH, Ecole française de Rome, 2000

Raymond, André, Grandes villes arabes d'époque ottomane, Paris, Sindbad, 1985

Réenchantement : http://reenchantement.free.fr

Signoles Pierre, El-Kadi Galila, Sidi Boumedine Rachid, (dir.), L'urbain dans le monde arabe. Politiques, instruments et acteurs, Paris, CNRS éditions, 1999

Troin, Jean-François, "Les villes du monde arabe: à la recherche d'un modèle », Bulletin de la société de géographie de Liège, 26, p. 53-62

Winkin, Yves, «Le touriste et son double. Eléments pour une anthropologie de l'enchantement », dans Ossman Susan, Miroirs Maghrébins, Paris, CNRS éditions, 1998

Ziadé, Khaled, Vendredi, dimanche, Paris, Actes Sud, 1996 